\title{
Inducing the human acrosome reaction with a calcium ionophore A23187 decreases sperm-zona pellucida binding with oocytes that failed to fertilize in vitro
}

\author{
D. Y. Liu and H. W. G. Baker
}

Department of Obstetrics and Gynaecology, University of Melbourne and Reproductive Biology Unit, Royal Women's Hospital, Melbourne, Victoria, Australia

\begin{abstract}
Summary. The human acrosome reaction was induced with the calcium ionophore A23187 and the proportion of reacted spermatozoa was determined with fluoresceinlabelled Pisum sativum agglutinin. Human oocytes that failed to fertilize in vitro were used to test binding of spermatozoa to the zona pellucida (ZP) and oolemma. Differential labelling of spermatozoa with fluorescein and rhodamine was used to control for variability in the oocytes. Spermatozoa labelled with one fluorochrome were treated with A23187 and mixed with equal numbers of motile untreated control spermatozoa labelled with the other fluorochrome. The mixture was incubated with zona-intact and zona-free oocytes for $4 \mathrm{~h}$. The sperm-ZP binding ratio of test to control spermatozoa was significantly decreased with increasing proportions of acrosome reacted spermatozoa. In contrast, the sperm-oolemma binding ratio was significantly increased with A23187 treatment. This suggests that acrosome-reacted spermatozoa do not bind to the human ZP.
\end{abstract}

Keywords: man; acrosome reaction; calcium ionophore; zona binding; oolemma binding

\section{Introduction}

In mammalian fertilization, spermatozoa must undergo the acrosome reaction (AR) to penetrate the zona pellucida (ZP) and fuse with the oolemma (Yanagimachi, 1981; Sathanathan et al., 1982; Wassarman, 1987a, b). Spermatozoa bound to or fused with the oolemma are therefore acrosomereacted. It is known that the relationship of the $A R$ and sperm $Z \mathrm{ZP}$ binding varies between species. In guinea-pigs, only acrosome-reacted spermatozoa bind to the ZP (Yanagimachi, 1981). In mice, only acrosome-intact spermatozoa bind to the $\mathrm{ZP}$ and the AR is induced later, probably by the interaction between sperm binding sites and one of the glycoproteins in the zona $\left(\mathbf{Z P}_{3}\right)$ (Wassarman, 1987a, b). Once mouse spermatozoa are acrosome-reacted, for example by exposure to the calcium ionophore A23187, they cannot bind to the ZP (Wassarman, 1987b). The situation in man is unclear because acrosome-intact and acrosome-reacted spermatozoa have been observed on the ZP (Overstreet \& Hembree, 1976). Although it is known that incubation of human spermatozoa in vitro to achieve capacitation will increase ZP binding (Singer et al., 1985), the relationship between the AR and sperm-ZP binding has not been studied in detail.

The present study was performed to determine whether, during fertilization in humans, the AR occurs before or after spermatozoa bind to the ZP. We used oocytes that failed to fertilize in a clinical in-vitro fertilization programme and A23187 to induce the AR. A23187-treated and control spermatozoa were labelled with different fluorochromes to control for variability of oocytes in their ability to bind spermatozoa (Liu et al., 1988a). Even though the A23187 is moderately effective in inducing the human AR, it decreases sperm motility. Therefore, we used equal concentrations 
progressively motile test and control spermatozoa in the incubation medium and checked for the possibility that immotile spermatozoa could bind to the oocytes (Aitken et al., 1983). If the AR occurs before sperm $-Z P$ binding, then the number of spermatozoa bound to the ZP should increase after treatment of spermatozoa with the A23187, whereas if spermatozoa bind to the ZP with the acrosome intact then inducing the AR should reduce the number of spermatozoa bound to the ZP.

\title{
Materials and Methods
}

\begin{abstract}
Human oocytes which failed to fertilize. Oocytes which showed no evidence of 2 pronuclei or cleavage $60 \mathrm{~h}$ after insemination in a clinical in-vitro fertilization programme were used for this study. The in-vitro fertilization procedures have been reported elsewhere (Liu et al., 1988b) and the project was approved by the Royal Women's Hospital Research and Ethics Committees. Some oocytes were kept in the incubator at $37^{\circ} \mathrm{C}$ in $5 \% \mathrm{CO}_{2}$ in air for an additional 1-2 days to accumulate enough oocytes for each experiment. Most $(60 \%)$ of the oocytes were obtained from patients for whom all the oocytes had failed to fertilize in vitro because of sperm defects. Most of the oocytes displayed regular shape and had lost the cumulus. In the remainder, the cumulus was removed by aspiration in and out of a glass micropipette. Morphological characteristics of these oocytes before insemination for in-vitro fertilization showed $40 \%$ good, $54 \%$ fair, and $6 \%$ poor using the criteria described previously (Nayudu et al., 1987).
\end{abstract}

Fluorochrome labelling of spermatozoa. Spermatozoa from 10 normal fertile donors were used for the experiments. The spermatozoa were labelled with fluorescein or rhodamine as described previously (Liu et al., 1988a). Labelling of spermatozoa with these two fluorochromes has no effect on sperm binding to the human ZP or fertilization in mice (Liu et al., 1988a). Briefly, $1 \mathrm{mg}$ fluorescein isothiocyanate (FITC: Sigma Chemical Co., St Louis, MO, USA) or $0.5 \mathrm{mg}$ tetramethylrhodamine B isothiocyanate (TRITC: Sigma) was dissolved in $0.1 \mathrm{ml}$ of $0.1 \mathrm{M}-\mathrm{KOH}$ and diluted within $15 \mathrm{sec}$ to $5 \mathrm{ml}$ with sterile Dulbecco phosphate-buffered saline (Commonwealth Serum Laboratory, Melbourne, Victoria, Australia) containing $55.5 \mathrm{~mm}$-glucose and stored at $4^{\circ} \mathrm{C}$ in a plastic tube wrapped in aluminium foil for up to 1 week. Sperm pellets were prepared by centrifuging $0.4 \mathrm{ml} \mathrm{semen} \mathrm{at} 600 \mathrm{~g}$ for $5 \mathrm{~min}$ to remove seminal plasma, and these were labelled by suspending them in $0.3 \mathrm{ml}$ of the fluorochrome solution. The suspension was incubated at $37^{\circ} \mathrm{C}$ for $15 \mathrm{~min}$ and the spermatozoa were recovered by centrifugation at $600 \mathrm{~g}$ for $5 \mathrm{~min}$. The sperm pellet was washed with $10 \mathrm{ml}$ Tyrode's solution (Commonwealth Serum Laboratories) with $0.2 \%$ bovine serum albumin (BSA) and after further centrifugation $(600 \mathrm{~g}, 10 \mathrm{~min})$ diluted to a concentration of $20 \times 10^{6} / \mathrm{ml}$ motile spermatozoa with human tubal fluid (HTF) medium (Quinn et al., 1985) containing $10 \%$ human serum inactivated by heating at $56^{\circ} \mathrm{C}$ for 30 min and sterilized by filtration using an Acrodisc filter $(0 \cdot 2 \mu \mathrm{m}$ : Gelman Sciences Inc., Ann Arbor, MI, USA).

Induction of the acrosome reaction. The fluorochrome-labelled spermatozoa were diluted to a concentration of $2 \times 10^{6}$ motile spermatozoa $/ \mathrm{ml}$. Spermatozoa labelled with one of the two fluorochromes were incubated with the A23187 (Sigma) for $\mathrm{l} \mathrm{h}$ at $37^{\circ} \mathrm{C}$ in $5 \% \mathrm{CO}_{2}$ in air. A $5 \mathrm{~mm}$ stock solution of A23187 in dimethyl sulphoxide (DMSO) was diluted 10 times with serum-free HTF medium before a small volume was added to the sperm suspension to achieve the required final concentration of A23187. Similar dilution was made for the DMSO control solution. Spermatozoa from the same donor but labelled with the other fluorochrome were used as controls and incubated with the same medium containing equivalent concentrations of DMSO. After incubation for $1 \mathrm{~h}$, the sperm suspensions were centrifuged, a sperm pellet obtained and the spermatozoa resuspended in HTF medium with $10 \%$ serum. Sperm motility was determined by phase-contrast microscopy.

Because A23187 reduces sperm motility the number of immotile spermatozoa in the test suspension was greater than that in the control suspension and increased with the concentration of A23187. The possibilities that the immotile spermatozoa present in the suspension could bind to the ZP or oolemma or influence the number of motile spermatozoa bound were investigated. Immotile spermatozoa were obtained by incubating TRITC-labelled spermatozoa $\left(2 \times 10^{6} / \mathrm{ml}\right)$ in medium containing $2.5 \%$ serum and $50 \mu \mathrm{M}-\mathrm{A} 23187$ for $1 \mathrm{~h}$. After incubation, the suspension was checked to confirm that all spermatozoa were immotile and the immotile spermatozoa were centrifuged and resuspended in HTF with $10 \%$ serum. To determine whether immotile spermatozoa could bind to the ZP or oolemma, a mixture of equal numbers of TRITC-labelled immotile spermatozoa treated with $50 \mu \mathrm{M}-\mathrm{A} 23187$ and FITC-labelled control spermatozoa ( $10^{5}$ motile and 0.35 to $0.45 \times 10^{5}$ immotile) was used.

Attempts to obtain high proportions of motile spermatozoa by the standard swim-up procedure after exposing the spermatozoa to A23187 were unsuccessful because of low yields. However, higher proportions of spermatozoa with progressive motility were obtained for test and control suspensions by a modified procedure: spermatozoa were labelled with FITC or TRITC and allowed to swim up into HTF medium containing A23187 or DMSO alone. HTF $(1.5 \mathrm{ml}$ ) containing $10 \%$ serum and $10 \mu \mathrm{M}-\mathrm{A} 23187$ or DMSO alone was carefully layered on the top of the FITC- and TRITC-labelled sperm suspensions respectively. After incubation for $1 \mathrm{~h}$ at $37^{\circ} \mathrm{C}, 1.0 \mathrm{ml}$ of the top layer of the suspension was aspirated, a sperm pellet was obtained by centrifugation $(600 \mathrm{~g}, 5 \mathrm{~min})$, and the pellet was resuspended in HTF with $10 \%$ serum. The final sperm motility was usually $15-20 \%$ lower in the A23187-exposed suspension than in the DMSO control. Equal numbers $\left(10^{5}\right)$ of motile test and control spermatozoa were mixed and the numbers of test and control immotile spermatozoa were equalized by addition of TRITC-labelled immotile spermatozga obtained 
by treatment with $50 \mu \mathrm{M}-\mathrm{A} 23187$. This mixture of spermatozoa therefore contained equal numbers of motile and immotile test and control spermatozoa.

To demonstrate that the AR had occurred in motile spermatozoa after A23187 treatment, $5 \mathrm{ml}$ suspensions of $2 \times 10^{6} / \mathrm{mi}$ FITC- or TRITC-labelled motile spermatozoa were treated separately with $10 \mu \mathrm{M}$-A23187 (test) or with DMSO (control) as described above. After incubation for $1 \mathrm{~h}$, the sperm suspensions were centrifuged, the supernatant removed and the sperm pellet resuspended in $0 \cdot 2-0.3 \mathrm{ml}$ HTF medium containing $20 \%$ serum and $5 \%$ BSA (pH 7.6). The concentrated sperm suspension was added to the bottom of a test tube $(15 \times 75 \mathrm{~mm})$ in which the inside wall of the tube had been wetted with the medium, then $1.5 \mathrm{ml}$ HTF medium containing $5 \%$ serum was carefully layered on top of the sperm suspension. After incubation for $1 \mathrm{~h}, 1-1.2 \mathrm{ml}$ of the top layer of the medium containing motile spermatozoa was aspirated. The motile sperm suspensions were centrifuged $(600 \mathrm{~g}, 7 \mathrm{~min})$, the supernatant removed and the sperm pellet resuspended in the fresh HTF containing $10 \%$ serum. This modified swim-up procedure using a discontinuous protein gradient between the sperm suspension and top layer of medium resulted in final progressive sperm motility percentages of $>90 \%$ in both test and control samples. While the mean percentage motility was not different between test and control samples, the total number of motile spermatozoa harvested was lower for the A23187-exposed spermatozoa. Equal numbers $\left(10^{5}\right)$ of motile test and control spermatozoa were mixed in $1 \mathrm{ml} \mathrm{HTF}$ containing $10 \%$ serum and incubated with 4 or $5 \mathrm{ZP}$.

Dose-response studies were performed with spermatozoa from 4 donors and A23187 concentrations between $1 \cdot 25$ and $20 \mu \mathrm{M}$. Oocytes were incubated with equal concentrations of motile A23187-treated and control spermatozoa but the concentrations of immotile spermatozoa were not adjusted as this did not affect sperm-ZP binding.

Acrosome assessment. Acrosome status was assessed on duplicate samples from A23187 and control incubations but the spermatozoa were not labelled with the fluorochromes. Fluorescein isothiocyanate-conjugated Pisum sativum agglutinin (FITC-PSA: Sigma) was used for detecting acrosome-intact or -reacted spermatozoa as described previously (Cross et al., 1986; Liu \& Baker, 1988). The proportion of spermatozoa with A23187-induced acrosome loss was calculated from the difference between the proportions without acrosomes in the control and A23187treated samples (\% induced AR $=\%$ acrosome-reacted spermatozoa incubated with A23187-\% acrosome-reacted spermatozoa incubated without A23187).

Sperm-oolemma binding test. The ZP of the oocytes was removed by brief exposure to acidic $0.9 \% \mathrm{NaCl}$ $(\mathrm{pH} 2 \cdot 5-3 \cdot 0$, with $\mathrm{HCl}$ ) with extensive aspiration in and out of a glass micropipette under observation with a dissecting microscope. When the ZP was removed the ZP-free oocyte was immediately transferred to HTF medium with $10 \%$ human serum and washed with 3-4 changes of the medium over 30-60 min. A mixture of equal numbers $\left(10^{5} / \mathrm{ml}\right.$ progressively motile spermatozoa) of test (incubated with A23187) and control (incubated without A23187) spermatozoa was added to $1-2$ ZP-free oocytes in $1 \mathrm{ml} \mathrm{HTF}$ medium with $10 \%$ human serum in a culture dish and the mixture stirred briefly with the pipette tip. Spermatozoa and ZP-free oocytes were incubated at $37^{\circ} \mathrm{C}$ in $5 \% \mathrm{CO}_{2}$ in air for $4 \mathrm{~h}$, then the oocytes were washed in 3-4 changes of Tyrode's solution with $0.2 \%$ BSA with extensive aspiration in and out of a glass micropipette to dislodge loosely adherent spermatozoa. Each ZP-free oocyte was then placed on a glass slide and flattened slightly with a coverslip supported at the edges with Vaseline. The number of spermatozoa bound on the oolemma was counted under a light fluorescence microscope (Dialux 20, Leitz, Wetzlar, West Germany) using excitation at $450-490 \mathrm{~nm}$ (filter No. 12) for FITC and $546 \mathrm{~nm}$ (filter No. 513690) for TRITC. The FITC-labelled spermatozoa fluoresced green and the TRITC-labelled spermatozoa red. The sperm-oolemma binding ratio was calculated from the total number of test spermatozoa divided by the total number of control spermatozoa bound to 5-9 ZP-free oocytes.

Sperm-ZP binding test. Methods used for the sperm-ZP binding test were as reported previously except that the oocytes were not stored in salt solutions (Liu et al., 1988a). A mixture of equal numbers ( $10^{5} / \mathrm{ml}$ progressively motile) of test and control spermatozoa, differentially labelled with the two fluorochromes, was incubated with the zonaintact oocytes for $4 \mathrm{~h}$. The oocytes were then washed in the medium to dislodge any loosely adherent spermatozoa and the numbers of spermatozoa labelled with each fluorochrome bound to the ZP were counted. The sperm-ZP binding ratio was calculated from the total number of test spermatozoa divided by the number of control spermatozoa bound to 5-8 zonae.

Statistical analysis. There were no significant departures from normality in the data. In the dose-response experiments, linear regression coefficients between concentration of the A23187, percentage induced AR, and sperm-ZP binding and sperm-oolemma binding ratios were calculated by the method of least squares. Multiple linear regression analysis was used to examine the effects of several factors including spermatozoa from different donors and dose of A23187 on zona and oolemma binding ratios.

\section{Results}

\section{The ability of spermatozoa immobilized by A23187 to bind to the ZP and oolemma}

When the mixed suspension of equal numbers of TRITC-labelled immotile spermatozoa obtained by treatment with $50 \mu \mathrm{M}-\mathrm{A} 23187$ and FITC-labelled control spermatozoa $\left(10^{5}\right.$ motile $_{2}$ and $\mathrm{g}_{2}$ 
35 or $45 \%$ immotile in 2 experiments) was incubated with the ZP-intact and ZP-free oocytes for $4 \mathrm{~h}$, there were no immotile spermatozoa bound on any of the $10 \mathrm{ZP}$ in contrast with a total of 39 control spermatozoa bound. However, there were 5 immotile spermatozoa bound on the oolemma from 11 ZP-free oocytes compared with a total of 44 motile control spermatozoa bound in the 2 experiments.

\section{Influence of immotile spermatozoa on the number of spermatozoa bound to the ZP and oolemma}

As shown in Table 1, when the numbers of motile and immotile spermatozoa were equalized for test (10 $\mu \mathrm{M}-\mathrm{A} 23187)$ and control (DMSO) treatments, and then incubated with the ZP-intact and the ZP-free oocytes, the number of test spermatozoa bound to the ZP was less than half that for control spermatozoa (mean ZP-binding ratio $=0.42$ ), but the number of test spermatozoa bound to the oolemma was double that of the control spermatozoa (mean oolemma-binding ratio $=2 \cdot 2$ ). Progressive motility averaged $55 \%$ for the test and $70 \%$ for the control sperm and the percentage induced AR (test-control) averaged $25 \%$.

Table 1. Effect of incubation with equal concentrations of motile and immotile spermatozoa on the number of spermatozoa bound to the ZP and oolemma

\begin{tabular}{cccccccc}
\hline & \multicolumn{3}{c}{ Sperm-ZP binding } & & \multicolumn{3}{c}{ Sperm-oolemma binding } \\
\cline { 2 - 4 } $\begin{array}{c}\text { Sperm } \\
\text { sample }\end{array}$ & $\begin{array}{c}\text { No. of } \\
\text { ZP }\end{array}$ & $\begin{array}{c}\text { Test/ } \\
\text { control* }\end{array}$ & Ratio & & $\begin{array}{c}\text { No. of } \\
\text { eggs }\end{array}$ & $\begin{array}{c}\text { Test/ } \\
\text { control* }\end{array}$ & Ratio \\
\hline A & 8 & $100 / 237$ & 0.42 & & $144 / 74$ & $2 \cdot 0$ \\
B & 5 & $14 / 34$ & 0.41 & & 5 & $124 / 60$ & $2 \cdot 1$ \\
C & 5 & $11 / 25$ & 0.44 & & 5 & $71 / 28$ & $2 \cdot 5$ \\
\hline
\end{tabular}

*Total no. of test spermatozoa/control spermatozoa bound.

\section{Acrosome reaction and ZP-binding capacity in A23187 treated motile spermatozoa}

Table 2 shows the percentage of induced AR and ZP-binding of the progressively motile spermatozoa selected by a modified swim-up procedure after exposing the spermatozoa to $10 \mu \mathrm{M}-$ A23187. The AR averaged $25 \%$ higher in the test than in the control spermatozoa. The mean ratio of test to control spermatozoa bound to the ZP was 0.43 for 3 experiments. There were similar percentages of motile test and control spermatozoa. The result is similar to the experiment reported in Table 1 in which there were higher proportions of immotile spermatozoa.

Table 2. Acrosome reaction (AR) and ZP-binding ability of motile spermatozoa selected by a modified swim-up procedure after incubating spermatozoa with $10 \mu \mathrm{M}-\mathrm{A} 23187$ (test, T) or DMSO (control, C)

\begin{tabular}{|c|c|c|c|c|c|}
\hline \multirow{2}{*}{$\begin{array}{l}\text { Sperm } \\
\text { sample }\end{array}$} & \multirow{2}{*}{$\frac{\text { Motility (\%) }}{\mathrm{T} / \mathrm{C}}$} & \multirow{2}{*}{$\frac{\operatorname{AR}(\%)}{T / C}$} & \multicolumn{3}{|c|}{ Sperm-ZP binding } \\
\hline & & & No. of ZP & $\mathrm{T} / \mathrm{C}^{*}$ & Ratio \\
\hline A & $97 / 97$ & $35 / 9$ & 10 & $49 / 145$ & $0 \cdot 34$ \\
\hline B & $90 / 93$ & $29 / 7$ & 12 & $51 / 86$ & 0.59 \\
\hline C & $93 / 96$ & $35 / 10$ & 10 & $36 / 100$ & 0.36 \\
\hline
\end{tabular}

*Total no. of test spermatozoa/control spermatozoa bound to $10_{\text {or }} 12 \mathrm{ZP}$ 


\section{Dose-response relationships between the AR induced by A23187, motility, and sperm-ZP and sperm-oolemma binding}

The average numbers of spermatozoa bound were $11 \cdot 2$ (range 0-200) per ZP and $12 \cdot 3$ (range 0-98) per ZP-free oocyte. The percentage of progressively motile spermatozoa decreased (Fig. 1) while the percentage of spermatozoa without an intact acrosome increased (Fig. 2) with increasing concentrations of A23187. Thus with $20 \mu \mathrm{M}-\mathrm{A} 23187$ there were on average $30 \%$ more acrosomereacted spermatozoa than with the control samples but sperm motility was severely reduced. Figure 2 shows that the sperm-ZP binding ratio decreased while the sperm-oolemma binding ratio increased with increasing concentrations of A23187. At $20 \mu \mathrm{M}-\mathrm{A} 23187$ the sperm-ZP binding ratio was halved and the sperm-oolemma binding ratio was trebled. At low concentrations of A23187 $(1 \cdot 25-5 \mu \mathrm{M})$, the increase in acrosome-reacted spermatozoa was $<10 \%$ and sperm-ZP binding ratio was unchanged but sperm-oolemma binding ratio was increased. At $10 \mu \mathrm{M}-\mathrm{A} 23187$ the results were similar to those shown in Tables 1 and 2.

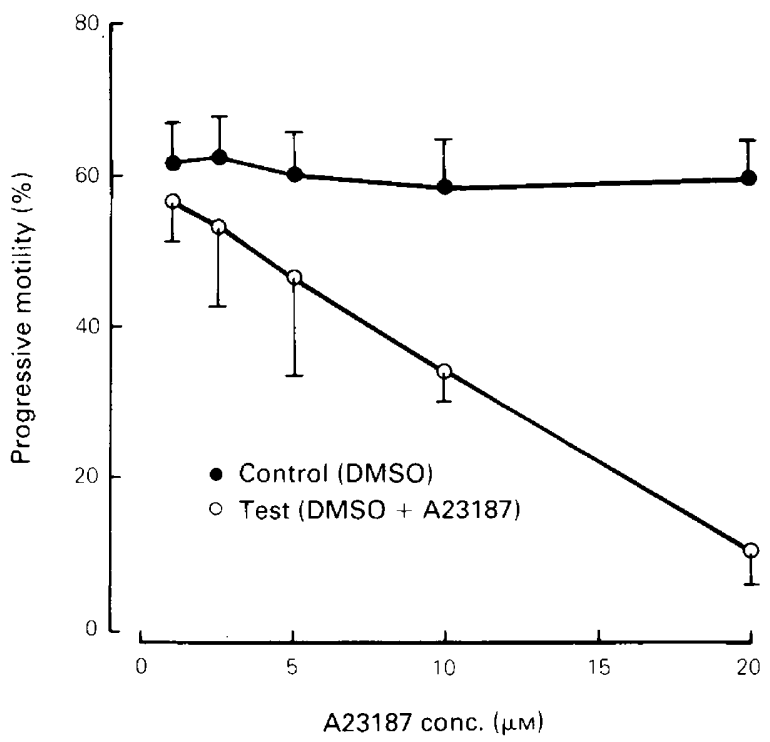

Fig. 1. Effect of the ionophore A23187 on human sperm motility; test spermatozoa were incubated with various concentrations of A23187; control spermatozoa were incubated with equivalent concentrations of DMSO. Linear regression analysis showed that motility of test spermatozoa was significantly decreased with increasing concentration of A23187 (regression coefficient $(b)=27 \cdot 529$, standard error (s.e. $)=3 \cdot 206, \mathrm{z}=8 \cdot 6, P<0 \cdot 001$ ).

Multiple linear regression analysis with percentage induced AR as the dependent variable showed a significant difference between donors in that the spermatozoa used in the second experiment had on average a lower percentage induced AR than did those used in the other experiments. Taking this difference between donors into account, $87.8 \%$ of the variance of percentage induced AR was explained by the regression on concentration of A23187. With sperm-ZP binding ratio as the dependent variable, the most significant explanatory variables were donor (the third donor having higher ratios than the other three) and percentage induced AR $\left(r^{2}=0.67\right)$. However, the sperm-oolemma binding ratio was more variable; regression analysis showed no differences between donors and the regression on concentration of A23187 $\left(r^{2}=0 \cdot 43\right)$ was more significant than that on percentage induced $\operatorname{AR}\left(r^{2}=0.36\right)$. 


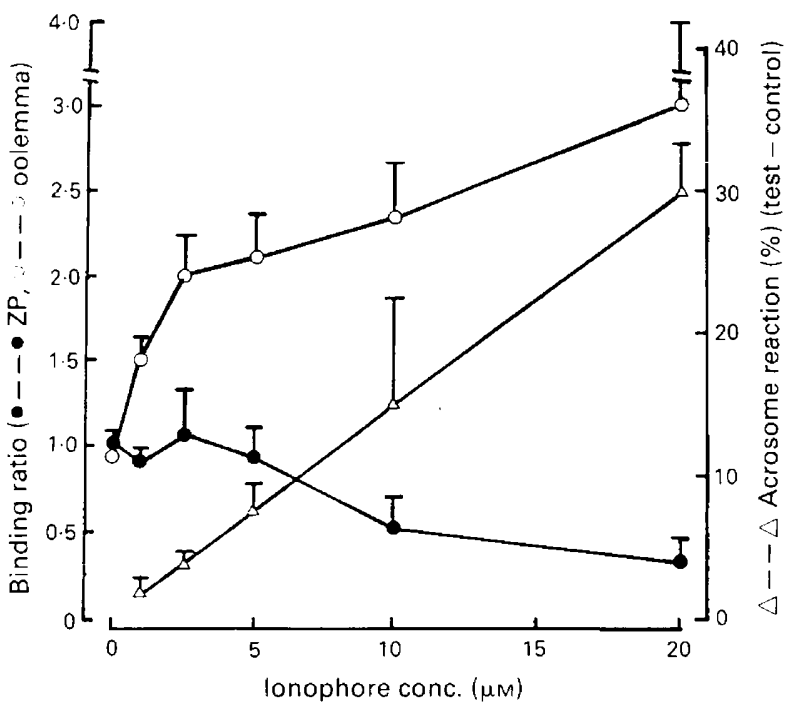

Fig. 2. Effect of the ionophore A23187 on sperm binding to the zona and oolemma. The sperm-ZP and sperm-oolemma binding ratios were calculated using a total of 80 (10-15 per point) ZP-intact and 61 (7-13 per point) ZP-free oocytes in 4 experiments with spermatozoa from different donors. The percentage induced acrosome reaction (AR) was calculated from the difference between proportions of test and control unlabelled spermatozoa without an acrosome determined with PSA-FITC. Linear regression coefficients on A23187 concentration were as follows: AR $(b=2 \cdot 175$, s.e. $=0.342, \mathrm{z}=6.4, P<0.001)$, ZP-binding ratio $(b=-0.393$, s.e. $=0.090, \mathrm{z}=4.4, P<0.001)$ and oolemma-binding ratio $(b=1 \cdot 113$, s.e. $=0 \cdot 294, \mathrm{z}=3 \cdot 8, P<0.01)$, and on AR for ZP-binding ratio $(b=-0.942$, s.e. $=0 \cdot 276$, $\mathrm{z}=3.4, P<0.01)$ and oolemma-binding ratio $(b=2.443$, s.e. $=0.904, \mathrm{z}=2.7, P<0.05)$.

\section{Discussion}

The present study shows that exposing spermatozoa to the calcium ionophore A23187 increased the number of acrosome-reacted spermatozoa and decreased the number of spermatozoa bound to the ZP. There was a significant inverse relationship between the number of spermatozoa bound to the ZP and the percentage of spermatozoa with induced AR. In addition, the number of spermatozoa bound to the oolemma increased. While A23187 is moderately effective in stimulating the AR in human spermatozoa it can cause a marked drop in sperm motility and so it was important in these experiments to incubate the oocytes with equal concentrations of progressively motile test and control spermatozoa. The decrease in the number of spermatozoa bound to the ZP was not likely to be due to the effect of the A23187 on motility since the number of progressively motile test and control spermatozoa was carefully equalized.

In the present study, we did not use a supravital stain or other method to assess sperm viability at the same time as the acrosome status was determined, because it is not possible to differentiate live motile and immotile spermatozoa. However, we isolated the motile spermatozoa by a modified swim-up procedure after exposing spermatozoa to A23187 and $>90 \%$ spermatozoa were progressively motile. The AR in A23187-treated spermatozoa was about $25 \%$ higher than for the control spermatozoa. Cross et al. (1986) have also shown that about 78\% acrosome-reacted spermatozoa are alive using supravital stain, Hoechst 33258, after exposure to A23187. Similar results were obtained for the ZP-binding ratios of these selected motile sperm suspensions. Even though we did not assess sperm velocity which may also play a role on sperm-ZP binding, we noted that the speed of progressive motility was not obviously different in the test and control suspensions by microscopy. The results of the dose-response experiments in which only motile spermatozoa were matched were the 
same as those for the preliminary experiments with matching of numbers of motile and immotile test and control spermatozoa or using samples with $>90 \%$ progressive motility.

Spermatozoa immobilized by exposure to $50 \mu \mathrm{M}$-A23187 did bind to the oolemma but only about $10 \%$ as well as did motile spermatozoa. Aitken et al. (1983) showed that immotile human spermatozoa were able to bind to zona-free hamster eggs. Under the present experimental conditions, we presume that some immotile acrosome-reacted spermatozoa may stick on the ZP-free oocytes as a result of mixing spermatozoa and oocytes in the culture dish during the addition of the spermatozoa. In the dose-response experiments, therefore, the difference in the number of immotile spermatozoa present in the test and control samples may have affected the oolemma-binding ratios slightly. However, the immotile spermatozoa did not bind to the ZP under the same conditions and did not alter the sperm-ZP binding ratios. The present results therefore indicate that increasing the proportion of acrosome-reacted spermatozoa in the medium decreases the number of spermatozoa bound to the ZP. This suggests that human spermatozoa initiate binding to the ZP with the acrosome intact; the AR then occurs during or after the process of zona binding. In this respect the human may be similar to the mouse and different from the guinea-pig (Wassarman, 1987a, b).

There is evidence that the human ZP plays a significant role in the induction of the AR (Cross et al., 1988; Aitken, 1989). The AR induced by A23187 is obviously different from the AR induced by the ZP in that the latter occurs on the zona and not in spermatozoa swimming free in medium. Cross et al. (1988) studied the time course of the AR of spermatozoa bound to the ZP and showed that the proportion of acrosome-reacted spermatozoa increased from $3 \%$ at $1 \mathrm{~min}$ to $46 \%$ during the next $60 \mathrm{~min}$. Accordingly, even though we do not know whether the initiation of the AR for the $3 \%$ spermatozoa occurred before or after binding to the ZP, it is obvious that the majority (97\%) of the spermatozoa bound to the ZP at an early stage were acrosome intact. Although solubilized human ZP material would be a more physiological inducer of the AR (Cross et al., 1988), the large number of human oocytes required for such experiments is difficult to obtain.

Exposure of spermatozoa to A23187 resulted in increased numbers of spermatozoa bound to the oolemma of ZP-free oocytes as expected. It is known that only acrosome-reacted spermatozoa are capable of binding to and fusing with zona-free hamster eggs (Koehler et al., 1982). Also, there is a positive correlation between the proportion of acrosome-reacted human spermatozoa and zona-free hamster egg penetration (Van Kooij et al., 1986; Yang et al., 1988). However, whether all spermatozoa bound to the oolemma are acrosome-reacted under the present experimental conditions is unknown. Furthermore, the sperm-oolemma binding ratio was more significantly related to the dose of A23187 than to the proportion of spermatozoa with an induced AR in the multiple regression analysis. It is therefore possible that the higher numbers of spermatozoa binding to the oolemma after treatment with A23187 may be augmented by mechanisms other than the higher proportion of motile acrosome-reacted spermatozoa in the medium. The ionophore may increase non-specific membrane adhesion and the interaction between the sperm and oocyte membrane after A23187 treatment may be unphysiological. Furthermore, with low concentrations of A23187 $(<5 \mu \mathrm{M})$ sperm-oolemma binding was increased without much effect on sperm motility. Also, numbers of acrosome-reacted spermatozoa were only slightly increased below this concentration. Despite the uncertainties about the mechanisms involved, this finding may be relevant to some new techniques that are being developed to assist fertilization in vitro for treatment of male infertility, including microinjection of human spermatozoa into the perivitelline space, zona drilling and zona opening (Gordon et al., 1988; Mann, 1988; Malter \& Cohen, 1989; Odawara \& Lopata, 1989). Because these techniques allow spermatozoa access to the oolemma without their binding to or penetration of the ZP it will probably be necessary to stimulate the AR to increase the chances of spermatozoa fusing with the oolemma. Unfortunately, A23187 is unsuitable for use in human in-vitro fertilization.

On the other hand increased proportions of acrosome-intact normal motile spermatozoa in insemination suspensions would be expected to improve success in standard in-vitro fertilization procedures. Liu et al. (1989) showed that the proportion of spermatozoa with a normal intact 
acrosome was significantly correlated with the number of spermatozoa bound to the ZP of oocytes unfertilized in in-vitro fertilization. We also found that the proportion of spermatozoa in the insemination medium with normal intact acrosomes was correlated with fertilization rates in vitro when sperm morphology was poor (Liu \& Baker, 1988). It is possible that spontaneous loss of acrosomes may be more frequent when spermatozoa have abnormal morphology and these spermatozoa may then be unable to bind to the ZP.

In summary, induction of the AR with the calcium ionophore A23187 decreased the number of spermatozoa bound to the ZP, suggesting that acrosome-reacted spermatozoa do not bind to the human ZP. Therefore, induction of the AR following binding to the ZP may be more important for the spermatozoon which ultimately fertilizes the human oocyte.

We thank all the scientists in the IVF laboratory for collecting the oocytes; and Gary N. Clarke for the sperm samples.

\section{References}

Aitken, R.J. (1989) Sperm-egg interaction. In Recent Advances in Andrology, pp. 52-62. Eds M. Serio \& G. Waites. Ares-Serono Symposia, Rome.

Aitken, R.J., Ross, A. \& Lees, M.M. (1983) Analysis of sperm function in Kartagener's syndrome. Fert. Steril. 40, 696698.

Cross, N.L., Morales, P., Overstreet, J.M. \& Hanson, F.W. (1986) Two simple methods for detecting acrosome-reacted human sperm. Gamete Res. 15, 213-226.

Cross, N.L., Morales, P., Overstreet, J.W. \& Hanson, F.W. (1988) Induction of acrosome reaction by the human zona pellucida. Biol. Reprod. 38, 235-244.

Gordon, J.W., Grunfeld, L., Garrisi, G.J., Talansky, B.E., Richards, C. \& Laufer, N. (1988) Fertilization of human oocytes by sperm from infertile males after zona pellucida drilling. Fert. Steril. 50, 68-73.

Koehler, J.K., De Curtis, I., Stenchever, A. \& Smith, D. (1982) Interaction of human sperm with zona free hamster eggs. A freeze-fracture study. Gamete Res. 6, 371-386.

Liu, D.Y. \& Baker, H.W.G. (1988) The proportion of sperm with poor morphology but normal intact acrosomes detected with pisum sativum agglutinin and fertilization in vitro. Fert. Steril. 50, 288-293.

Liu, D.Y., Lopata, L., Johnston, W.I.H. \& Baker, H.W.G. (1988a) A human sperm-zona pellucida binding test using oocytes that failed to fertilize in vitro. Fert. Steril. 50, 782-788.

Liu, D.Y., Du Plessis, Y.P., Nayudu, P.L., Johnston, W.I.H. \& Baker, H.W.G. (1988b) The use of in vitro fertilization to evaluate putative tests of human sperm function. Fert. Steril. 49, 272-277.

Liu, D.Y., Lopata, L., Johnston, W.I.H. \& Baker, H.W.G. (1989) Human sperm-zona pellucida binding, sperm characteristics and fertilization in vitro. Human Reprod. 4, 396-701.

Malter, H.M. \& Cohen, J. (1989) Partial zona dissection of the human oocyte: a nontraumatic method using micromanipulation to assist zona pellucida penetration. Fert. Steril. 51, 139-148.

Mann, J.R. (1988) Full term development of mouse eggs fertilized by a spermatozoon microinjected under the zona pellucida. Biol. Reprod. 38, 1077-1083.
Nayudu, P.L., Gook, D.A., Lopata, A., Sheather, S.J., Lloyd-Smith, C.W., Cadusch, P. \& Johnston, W.I.H. (1987) Follicular characteristics associated with viable pregnancy after in vitro fertilization in humans. Gamete Res. 18, 37-55.

Odawara, Y. \& Lopata, A. (1989) A zona opening procedure for improving in vitro fertilization at low sperm concentrations: a mouse model. Fert. Steril. 51, 699-704.

Overstreet, J.W. \& Hembree, W.C. (1976) Penetration of the zona pellucida of nonliving human oocytes by human spermatozoa in vitro. Fert. Steril. 27, 815-831.

Quinn, P., Kerin, J.F. \& Warnes, G.M. (1985) Improved pregnancy rate in human vitro fertilization with the use of a medium based on the composition of human tubal fluid. Fert. Steril. 44, 493-498.

Sathananthan, A.H., Trounson, A.O., Wood, C. \& Leeton, J.F. (1982) Ultrastructural observation on the penetration of human sperm into the zona pellucida of the human egg in vitro. $J$. Androl. 3, 356-364.

Singer, S.L., Lambert, H., Overstreet, J.W., Hanson, F.W. \& Yanagimachi, R. (1985) The kinetics of human sperm binding to the human zona pellucida and zona-free hamster oocyte in vitro. Gamete Res. 12, 29-39.

Van Kooij, R.J., Balerna, M., Roatti, A. \& Campana, A. (1986) Oocytes penetration and acrosome reaction of human spermatozoa. I. Influence of incubation time and medium composition. Andrologia 18, 152-160.

Wassarman, P.M. (1987a) Early events in mammalian fertilization. Ann. Rev. Cell Biol. 3, 109-142.

Wassarman, P.M. (1987b) The biology and chemistry of fertilization. Science, $N Y$ 235, 553-560.

Yanagimachi, R. (1981) Mechanisms of fertilization in mammals. In Fertilization and Embryonic Development in Vitro, pp. 81-182. Eds L. Mastroianni \& J. D. Biggers. Plenum Press, New York.

Yang, S.Y., Rojas, F.J. \& Stone, S.C. (1988) Acrosome reaction of human spermatozoa in zona-free hamster egg penetration test. Fert. Steril. 50, 954-959. 